\title{
On a Hyperplane Arrangement Problem and Tighter Analysis of an Error-Tolerant Pooling Design
}

\author{
Hung Q. Ngo *
}

August 19, 2006

\begin{abstract}
In this paper, we formulate and investigate the following problem: given integers $d, k$ and $r$ where $k>r \geq 1, d \geq 2$, and a prime power $q$, arrange $d$ hyperplanes on $\mathbb{F}_{q}^{k}$ to maximize the number of $r$-dimensional subspaces of $\mathbb{F}_{q}^{k}$ each of which belongs to at least one of the hyperplanes. The problem is motivated by the need to give tighter bounds for an error-tolerant pooling design based on finite vector spaces.
\end{abstract}

\section{Introduction}

Designing good error-tolerant pooling design is a central problem in the area of non-adaptive group testing [9], which has many practical applications including DNA library screening [8, 10,21], multiple access control [5-7, 17,26], and error correcting/detecting superimposed codes [11-15], to name a few.

To date, there are relatively few papers addressing the problem of designing and analyzing errortolerant pooling designs $[1,3,4,16,19,20,22,24]$. In [22], Ngo and Du introduced a non-adaptive pooling design based on finite vector spaces, which was later found to be highly error-tolerant by D'yachkov et a. [10]. The analysis of the design in [10] was not very tight. In this paper, we give a tighter analysis of the design. This is done via formulating a new and very interesting hyperplane arrangement problem on finite fields.

To formally describe our problem, we first need a few definitions. A 01-matrix $M$ is said to be $d$-disjunct if and only if no column is contained in the union of $d$ others. (Here, columns are viewed as characteristic vectors of sets of rows.) A $d$-disjunct matrix corresponds precisely to a pooling design which can identify at most $d$ negative items. For the design to tolerate a few errors in outcomes, it is not sufficient for a column to just not be covered by $d$ others. A $d^{z}$-disjunct matrix is a matrix where, given any $d+1$ columns $C_{0}, C_{1}, \ldots, C_{d}$, the set $C_{0} \backslash C_{1} \cup \cdots \cup C_{d}$ has at least $z$ elements. It is easy to show that a $d^{z}$-disjunct matrix can detect $z-1$ errors and correct $\lfloor(z-1) / 2\rfloor$ errors.

The construction in [22] is as follows. Let $q$ be a prime power and $m, k, r$ be integers such that $m>k>r \geq 1$. Let $M_{q}(m, k, r)$ be the 01 -matrix whose rows are indexed by $r$-dimensional subspaces of $\mathbb{F}_{q}^{m}$ and whose columns are indexed by $k$-dimensional subspaces of $\mathbb{F}_{q}^{m} . M_{q}(m, k, r)$ has a 1 in row $R$ and column $C$ if and only if $R$ is a subspace of $C$. It is easy to see that $M_{q}(m, k, d)$ is $d$-disjunct (the containment method by Macula [18]). Later, D'yachkov et al. [10] realized that we do not have to take $r=d$ for $M_{q}(m, k, r)$ to be $d$-disjunct ( $r$ could be a lot smaller than $d$, even $r=1$ works sometimes). Moreover, the construction can, in general, tolerate a lot of errors. Specifically, their main result was that,

\footnotetext{
${ }^{*}$ Computer Science and Engineering Department, SUNY at Buffalo, Amherst, NY 14260, USA. E-mail: hungngo@cse. buffalo. edu. This work is partially supported by NSF CAREER Award CCF-0347565.
} 
for any $d+1 k$-dimensional subspaces $C_{0}, C_{1}, \ldots, C_{d}$ of $\mathbb{F}_{q}^{m}$, the number of $r$-dimensional subspaces each of which belongs to $C_{0}$ but not other $C_{i}$ is at least

$$
\left[\begin{array}{l}
k \\
r
\end{array}\right]_{q}-d\left[\begin{array}{c}
k-1 \\
r
\end{array}\right]_{q}+(d-1)\left[\begin{array}{c}
k-2 \\
r
\end{array}\right]_{q}
$$

and that the bound is tight for $d \leq q+1$. Here, for any non-negative integers $m, n,\left[\begin{array}{c}n \\ m\end{array}\right]_{q}$ denotes the Gaussian coefficient, to be defined in the next section.

The number of columns of $M_{q}(m, k, r)$ is $\left[\begin{array}{c}m \\ k\end{array}\right]_{q}$, exponentially larger than $q+1$. Hence, it is desirable to devise tight bounds for the case when $q+1<d \leq\left[\begin{array}{c}m \\ k\end{array}\right]_{q}-1$. In this paper, we partially address this problem. In the process, we formulate a new - to the best of the author's knowledge - hyperplane arrangement problem on finite fields.

The rest of this paper is organized as follows. Section 2 motivates the hyperplane arrangement problem and presents preliminary results on the problem. Section 3 gives tighter bounds for the original group testing problem using results from Section 2. Section 4 concludes the paper with additional remarks and a conjecture.

\section{An extremal hyperplane arrangement problem on finite fields}

\subsection{Motivation and notations}

Henceforth, we shall use $(a ; q)_{n}$ (or $(a)_{n}$ for short) to denote the $q$-shifted factorial:

$$
(a)_{n}=(a ; q)_{n}:=(1-a)(1-a q) \ldots\left(1-a q^{n-1}\right) .
$$

The $q$-analogue of a natural number $n$ is denoted by $[n]_{q}$, and the Gaussian coefficient is denoted by $\left[\begin{array}{l}n \\ m\end{array}\right]_{q}$. They are defined as follows.

$$
\begin{aligned}
{[0]_{q} } & :=0 \\
{[n]_{q} } & :=1+q+\cdots+q^{n-1}, n \geq 1 \\
{\left[\begin{array}{c}
n \\
m
\end{array}\right]_{q} } & := \begin{cases}0 & \text { when } n<m \\
\frac{(q)_{n}}{(q)_{n-m}(q)_{m}}=\frac{\left(1-q^{n}\right) \ldots\left(1-q^{n-m+1}\right)}{\left(1-q^{m}\right) \ldots(1-q)} & \text { otherwise. }\end{cases}
\end{aligned}
$$

We shall drop the subscript $q$ and write $[n]$ and $\left[\begin{array}{c}n \\ m\end{array}\right]$ when there is no potential confusion as to what $q$ is. Our notations are standard in the $q$-series literature [2].

For any vector space $X$, let $\bar{X}$ denote the set of all $r$-dimensional subspaces of $X$, and $\operatorname{dim}(X)$ the dimension of $X$. Then, it is well known (see, e.g. [25]) that

$$
|\bar{X}|=\left[\begin{array}{c}
\operatorname{dim}(X) \\
r
\end{array}\right] \text {. }
$$

For any vector spaces $X$ and $Y$,

$$
\bar{X} \cap \bar{Y}=\overline{X \cap Y}
$$

because any vector space which is a subspace of $X$ and a subspace of $Y$ is also a subspace of the vector space $X \cap Y$. Note that, in general $X \cup Y$ is not a vector space, and

$$
\bar{X} \cup \bar{Y} \neq \overline{\operatorname{span}(X \cup Y)} \text {. }
$$

The matrix $M_{q}(m, k, r)$ is $d^{z}$-disjunct for

$$
z=\min \left\{\left|\overline{C_{0}} \backslash \overline{C_{1}} \cup \cdots \cup \overline{C_{d}}\right|: C_{0}, C_{1}, \ldots C_{d} \text { are } d+1 \text { different } k \text {-dimensional subspaces of } \mathbb{F}_{q}^{m}\right\} \text {. }
$$


Thus, we want to find $k$-dimensional subspaces $C_{0}, C_{1}, \ldots, C_{d}$ of $\mathbb{F}_{q}^{m}$ that minimizes the quantity

$$
\left|\overline{C_{0}} \backslash \overline{C_{1}} \cup \cdots \cup \overline{C_{d}}\right| .
$$

For any $i \in\{1, \ldots, d\}$, let $H_{i}=C_{i} \cap C_{0}$, then

$$
\begin{aligned}
\left|\overline{C_{0}} \backslash \overline{C_{1}} \cup \cdots \cup \overline{C_{d}}\right| & =\left|\overline{C_{0}} \backslash\left(\overline{C_{1}} \cap \overline{C_{0}}\right) \cup \cdots \cup\left(\overline{C_{d}} \cap \overline{C_{0}}\right)\right| \\
& =\left|\overline{C_{0}} \backslash\left(\overline{C_{1} \cap \overline{C_{0}}}\right) \cup \cdots \cup\left(\overline{C_{d} \cap \overline{C_{0}}}\right)\right| \\
& =\left|\overline{C_{0}} \backslash \overline{H_{1}} \cup \cdots \cup \overline{H_{d}}\right| .
\end{aligned}
$$

For $\left|\overline{C_{0}} \backslash \overline{C_{1}} \cup \cdots \cup \overline{C_{d}}\right|$ to be minimized, we can assume that all $H_{i}$ are hyperplanes of $C_{0}$. The number of hyperplanes of $C_{0}$ is $\left[\begin{array}{c}k \\ k-1\end{array}\right]=[k]$. Thus, when $d \leq[k]$ we can also assume that the $H_{i}$ are different hyperplanes of $C_{0}$; because, given $d$ hyperplanes $H_{1}, \ldots, H_{d}$, we can take the span of each of them with a vector $v \notin C_{0}$ to reconstruct the $C_{i}$. For the group testing problem, we will address the case when $d>[k]$ in a later section. In this section, we only consider the case when $d \leq[k]$. Because $\left|\overline{C_{0}} \backslash \overline{C_{1}} \cup \cdots \cup \overline{C_{d}}\right|$ is minimized when $\left|\overline{H_{1}} \cup \cdots \cup \overline{H_{d}}\right|$ is maximized, the above discussion motivates the following problem.

Problem 1 (Our Hyperplane Arrangement Problem). Given a $k$-dimensional vector space $C$ over $\mathbb{F}_{q}$, and an integer $d$ such that $1 \leq d \leq[k]$, find $d$ hyperplanes $H_{1}, \ldots, H_{d}$ of $C$ that maximizes the following quantity

$$
\left|\overline{H_{1}} \cup \cdots \cup \overline{H_{d}}\right| .
$$

At least, find good upper bounds for the quantity.

The result in [10] can be restated as follows

Theorem 2.1 (D'yachkov et al.). Given integers $r \leq k-2$, and $d \leq[k]$. Let $H_{1}, \ldots, H_{d}$ be $d$ different hyperplanes of a $k$-dimensional vector space over $\mathbb{F}_{q}$. Then,

$$
\left|\overline{H_{1}} \cup \cdots \cup \overline{H_{d}}\right| \leq d\left[\begin{array}{c}
k-1 \\
r
\end{array}\right]-(d-1)\left[\begin{array}{c}
k-2 \\
r
\end{array}\right]
$$

The bound is tight when $d \leq q+1$.

\subsection{Initial observations}

By inclusion-exclusion, we have

$$
\begin{aligned}
& \left|\overline{H_{1}} \cup \cdots \cup \overline{H_{d}}\right|=\sum_{i=1}^{d}(-1)^{i-1} \sum_{\substack{T \subseteq\{1, \ldots, d\} \\
|T|=i}} \bigcap_{t \in T} \overline{H_{t}} \\
& =\sum_{i=1}^{d}(-1)^{i-1} \sum_{\substack{T \subseteq\{1, \ldots, d\} \\
|T|=i}} \overline{\bigcap_{t \in T} H_{t}} \\
& =\sum_{i=1}^{d}(-1)^{i-1} \sum_{\substack{T \subseteq\{1, \ldots, d\} \\
|T|=i}}\left[\begin{array}{c}
\operatorname{dim}\left(\bigcap_{t \in T} H_{t}\right) \\
r
\end{array}\right]
\end{aligned}
$$

As we will see later, it is not easy to determine the dimension of the intersection of a given number of arbitrary hyperplanes. That is why inclusion-exclusion does not help us directly solve the problem. Next, for any two vector spaces $X$ and $Y$,

$$
\operatorname{dim}(X)+\operatorname{dim}(Y)=\operatorname{dim}(\operatorname{span}(X \cup Y))+\operatorname{dim}(X \cap Y) .
$$


In particular, if $X$ is a hyperplane and $Y$ is a proper subspace of a $k$-dimensional vector space, then either $Y \subseteq X$ or $\operatorname{dim}(Y)=\operatorname{dim}(X \cap Y)+1$. To see this, suppose $Y$ has dimension $l \leq k-1$. If $Y \nsubseteq X$, then $\operatorname{dim}(\operatorname{span}(X \cup Y))=k$, which implies

$$
\operatorname{dim}(Y)=k+\operatorname{dim}(X \cap Y)-\operatorname{dim}(X)=\operatorname{dim}(X \cap Y)+1 .
$$

In words, a hyperplane either contains $Y$ or "cut into" $Y$ at one dimension lower than that of $Y$. This observation leads to the following simple yet important lemma.

Lemma 2.2. Let $H_{1}, \ldots, H_{x}$ be some $x$ hyperplanes of an l-dimensional vector space over $\mathbb{F}_{q}$ whose intersection is $I=H_{1} \cap \cdots \cap H_{x}$. Let $H$ be any hyperplane not containing $I$, and set $Y_{i}=H \cap H_{i}$, $i \in\{1, \ldots, x\}$. Then, for any subset $S \subseteq\{1, \ldots, x\}$, we have

$$
\operatorname{dim}\left(\bigcap_{i \in S} H_{i}\right)=\operatorname{dim}\left(\bigcap_{i \in S} Y_{i}\right)+1
$$

Proof. Because $H$ does not contain $I, H$ does not contain $\bigcap_{i \in S} H_{i}$ for any $S$. Thus,

$$
\operatorname{dim}\left(\bigcap_{i \in S} H_{i}\right)=\operatorname{dim}\left(H \cap \bigcap_{i \in S} H_{i}\right)+1=\operatorname{dim}\left(\bigcap_{i \in S}\left(H_{i} \cap H\right)\right)+1=\operatorname{dim}\left(\bigcap_{i \in S} Y_{i}\right)+1
$$

The $Y_{i}$ actually are hyperplanes of $H$. What this lemma tells us is that, the inter-relationship (in terms of dimensions of intersections) between the hyperplanes $H_{1}, \ldots, H_{x}$ is the same as the inter-relationship between the hyperplanes $Y_{1}, \ldots, Y_{x}$ of $H$. The hyperplanes $Y_{1}, \ldots, Y_{x}$ form a down-scaled picture of $H_{1}, \ldots, H_{x}$ inside $H$.

Consider an $i$-dimensional subspace $X$ of a $k$-dimensional vector space $S$ over $\mathbb{F}_{q}$. Let $l$ be an integer where $i \leq l \leq k$. Then, the number of $l$-dimensional subspaces of $S$ containing $X$ is $\left[\begin{array}{c}k-i \\ l-i\end{array}\right]$. In particular, when $i=k-2$ the number of hyperplanes that contains $X$ is

$$
\left[\begin{array}{c}
k-(k-2) \\
k-1-(k-2)
\end{array}\right]=\left[\begin{array}{l}
2 \\
1
\end{array}\right]=q+1 .
$$

Lastly, the following identity is the $q$-analog of the Pascal's triangle identity for binomial coefficients [25]:

$$
\left[\begin{array}{c}
n \\
m
\end{array}\right]_{q}=\left[\begin{array}{c}
n-1 \\
m
\end{array}\right]_{q}+q^{n-m}\left[\begin{array}{c}
n-1 \\
m-1
\end{array}\right]_{q}
$$

\subsection{The cases of 4 and 5 hyperplanes}

Using the basic observations in the previous section, when there are a constant number of hyperplanes it is possible to enumerate all possible classes of arrangements (with respect to our objective function). In this section, we will compute the objective function for all arrangements of 4 and 5 hyperplanes. These arrangements will serve as the base case to prove generic bounds in the next section.

We will be working on an $l$-dimensional vector space $S$ over $\mathbb{F}_{q}$, namely $S$ is isomorphic to $\mathbb{F}_{q}^{l}$. For any set of (at least two) hyperplanes $\mathcal{H}$, let $x(\mathcal{H})$ be the maximum number of hyperplanes in $\mathcal{H}$ whose intersection has dimension $l-2$. Note that $2 \leq x(\mathcal{H}) \leq q+1$. Also define

$$
g(\mathcal{H})=\left|\bigcup_{H \in \mathcal{H}} \bar{H}\right|
$$

We first consider the 4-hyperplane case. 
Lemma 2.3. Let $\mathcal{H}=\left\{H_{1}, H_{2}, H_{3}, H_{4}\right\}$ be a set of 4 hyperplanes of $\mathbb{F}_{q}^{l}$.

(i) If $x(\mathcal{H})=4$, then

$$
g(\mathcal{H})=g_{1}^{(4)}:=4\left[\begin{array}{c}
l-1 \\
r
\end{array}\right]-3\left[\begin{array}{c}
l-2 \\
r
\end{array}\right] .
$$

This case can only hold when $q \geq 3$.

(ii) If $x(\mathcal{H})=3$, then

$$
g(\mathcal{H})=g_{2}^{(4)}:=4\left[\begin{array}{c}
l-1 \\
r
\end{array}\right]-5\left[\begin{array}{c}
l-2 \\
r
\end{array}\right]+2\left[\begin{array}{c}
l-3 \\
r
\end{array}\right]
$$

(iii) If $x(\mathcal{H})=2$, then there are two cases:

$$
\begin{aligned}
& g(\mathcal{H})=g_{3}^{(4)}:=4\left[\begin{array}{c}
l-1 \\
r
\end{array}\right]-6\left[\begin{array}{c}
l-2 \\
r
\end{array}\right]+4\left[\begin{array}{c}
l-3 \\
r
\end{array}\right]-\left[\begin{array}{c}
l-4 \\
r
\end{array}\right] \\
& g(\mathcal{H})=g_{4}^{(4)}:=4\left[\begin{array}{c}
l-1 \\
r
\end{array}\right]-6\left[\begin{array}{c}
l-2 \\
r
\end{array}\right]+3\left[\begin{array}{c}
l-3 \\
r
\end{array}\right] .
\end{aligned}
$$

Moreover, $g_{1}^{(4)} \geq g_{2}^{(4)} \geq g_{3}^{(4)} \geq g_{4}^{(4)}$.

Proof. Cases $(i)$ and (ii) follow straightforwardly from the inclusion-exclusion formula (3) and Lemma 2.2. Suppose $x(\mathcal{H})=2$, then $W=H_{1} \cap H_{2} \cap H_{3}$ has dimension $l-3$. If $H_{4}$ does not contain $W$ then $H_{1} \cap H_{2} \cap H_{3} \cap H_{4}$ has dimension $l-4$, and the formula for $g_{3}^{(4)}$ follows from (3) again. (Note that, when $l \leq 3$ all formulas follow trivially.) Thus, the last case is when $W \subset H_{4}$. Let $V_{i}=H_{4} \cap H_{i}$, for $i=1,2,3$. Because $x(\mathcal{H})=2$, the $V_{i}$ are three different hyperplanes of $H_{4}$. Moreover, $V_{1} \cap V_{2} \cap V_{3}=W$, and $\operatorname{dim}\left(V_{i}\right)=l-2$ for $i=1,2,3$. We can compute $g(\mathcal{H})$ as follows, noting Lemma 2.2,

$$
\begin{aligned}
g(\mathcal{H}) & =\left|\overline{H_{1}} \cup \overline{H_{2}} \cup \overline{H_{3}}\right|+\left|\overline{H_{4}} \backslash \overline{H_{1}} \cup \overline{H_{2}} \cup \overline{H_{3}}\right| \\
& =\left|\overline{H_{1}} \cup \overline{H_{2}} \cup \overline{H_{3}}\right|+\left|\overline{H_{4}} \backslash \overline{V_{1}} \cup \overline{V_{2}} \cup \overline{V_{3}}\right| \\
& =\left|\overline{H_{1}} \cup \overline{H_{2}} \cup \overline{H_{3}}\right|+\left|\overline{H_{4}}\right|-\left|\overline{V_{1}} \cup \overline{V_{2}} \cup \overline{V_{3}}\right| \\
& =\left(3\left[\begin{array}{c}
l-1 \\
r
\end{array}\right]-3\left[\begin{array}{c}
l-2 \\
4
\end{array}\right]+\left[\begin{array}{c}
l-3 \\
r
\end{array}\right]\right)+\left[\begin{array}{c}
l-1 \\
r
\end{array}\right]-\left(3\left[\begin{array}{c}
l-2 \\
r
\end{array}\right]-2\left[\begin{array}{c}
l-3 \\
r
\end{array}\right]\right) \\
& =g_{4}^{(4)} .
\end{aligned}
$$

Lemma 2.4. Let $\mathcal{H}=\left\{H_{1}, H_{2}, H_{3}, H_{4}, H_{5}\right\}$ be a set of 5 hyperplanes of $\mathbb{F}_{q}^{l}$.

(i) If $x(\mathcal{H})=5$, then

$$
g(\mathcal{H})=g_{1}^{(5)}:=5\left[\begin{array}{c}
l-1 \\
r
\end{array}\right]-4\left[\begin{array}{c}
l-2 \\
r
\end{array}\right] .
$$

This case can only hold when $q \geq 4$.

(ii) If $x(\mathcal{H})=4$, then

$$
g(\mathcal{H})=g_{2}^{(5)}:=5\left[\begin{array}{c}
l-1 \\
r
\end{array}\right]-7\left[\begin{array}{c}
l-2 \\
r
\end{array}\right]+3\left[\begin{array}{c}
l-3 \\
r
\end{array}\right] .
$$

This case can only hold when $q \geq 3$. 
(iii) If $x(\mathcal{H})=3$, then there are three cases:

$$
\begin{aligned}
& g(\mathcal{H})=g_{3}^{(5)}:=5\left[\begin{array}{c}
l-1 \\
r
\end{array}\right]-8\left[\begin{array}{c}
l-2 \\
r
\end{array}\right]+4\left[\begin{array}{c}
l-3 \\
r
\end{array}\right] \\
& g(\mathcal{H})=g_{4}^{(5)}:=5\left[\begin{array}{c}
l-1 \\
r
\end{array}\right]-9\left[\begin{array}{c}
l-2 \\
r
\end{array}\right]+7\left[\begin{array}{c}
l-3 \\
r
\end{array}\right]-2\left[\begin{array}{c}
l-4 \\
r
\end{array}\right], \\
& g(\mathcal{H})=g_{5}^{(5)} \quad:=5\left[\begin{array}{c}
l-1 \\
r
\end{array}\right]-9\left[\begin{array}{c}
l-2 \\
r
\end{array}\right]+5\left[\begin{array}{c}
l-3 \\
r
\end{array}\right] .
\end{aligned}
$$

The last case (of $g_{5}^{(5)}$ ) can only hold when $q \geq 3$ ).

(iv) If $x(\mathcal{H})=2$, then there are four cases:

$$
\begin{aligned}
g(\mathcal{H})=g_{6}^{(5)} & :=5\left[\begin{array}{c}
l-1 \\
r
\end{array}\right]-10\left[\begin{array}{c}
l-2 \\
r
\end{array}\right]+10\left[\begin{array}{c}
l-3 \\
r
\end{array}\right]-4\left[\begin{array}{c}
l-4 \\
r
\end{array}\right], \\
g(\mathcal{H})=g_{7}^{(5)} & :=5\left[\begin{array}{c}
l-1 \\
r
\end{array}\right]-10\left[\begin{array}{c}
l-2 \\
r
\end{array}\right]+10\left[\begin{array}{c}
l-3 \\
r
\end{array}\right]-5\left[\begin{array}{c}
l-4 \\
r
\end{array}\right]+\left[\begin{array}{c}
l-5 \\
r
\end{array}\right], \\
g(\mathcal{H})=g_{8}^{(5)} & :=5\left[\begin{array}{c}
l-1 \\
r
\end{array}\right]-10\left[\begin{array}{c}
l-2 \\
r
\end{array}\right]+9\left[\begin{array}{c}
l-3 \\
r
\end{array}\right]-3\left[\begin{array}{c}
l-4 \\
r
\end{array}\right], \\
g(\mathcal{H})=g_{9}^{(5)} & :=5\left[\begin{array}{c}
l-1 \\
r
\end{array}\right]-10\left[\begin{array}{c}
l-2 \\
r
\end{array}\right]+6\left[\begin{array}{c}
l-3 \\
r
\end{array}\right] .
\end{aligned}
$$

The last case (of $g_{9}^{(5)}$ can only hold when $q \geq 3$ ).

Moreover, $g_{1}^{(5)} \geq g_{2}^{(5)} \geq g_{3}^{(5)} \geq g_{4}^{(5)} \geq g_{5}^{(5)}$; and $g_{4}^{(5)} \geq g_{6}^{(5)} \geq g_{7}^{(5)} \geq g_{8}^{(5)} \geq g_{9}^{(5)}$. Also, $g_{5}^{(5)} \geq g_{6}^{(5)}$ when $q \geq 4$.

Proof. $\quad$ Cases $(i)$ and $(i i)$ follow straightforwardly from the inclusion-exclusion formula (3) and Lemma 2.2.

- Suppose $x(\mathcal{H})=3$, and assume $V=H_{1} \cap H_{2} \cap H_{3}$ has dimension $l-2$. Let $V_{i}=H_{4} \cap H_{i}$, for $i=1,2,3$, and $U=H_{1} \cap H_{2} \cap H_{3} \cap H_{4}$. Since $x(\mathcal{H})=3, H_{4}$ does not contain $V$ and thus $\operatorname{dim}(U)=l-3$ by Lemma (2.2). We consider three cases as follows.

Case 1: $H_{5}$ contains some $V_{i}$ for $i=1,2,3$. Note that $H_{5}$ cannot contain two different $V_{i}$ because the span of two different $V_{i}$ is exactly $H_{4}$. Without loss of generality, assume $V_{1} \subset H_{5}$. In this case $H_{1}, H_{2}, H_{3}, H_{4}$ intersect $H_{5}$ at 3 different hyperplanes (of $H_{5}$ ), because $H_{1}$ and $H_{4}$ intersect $H_{5}$ at the same hyperplane $V_{1}$. Lemma 2.2 and the inclusion-exclusion formula (3) gives

$$
\begin{aligned}
\left|\overline{H_{1}} \cup \cdots \cup \overline{H_{5}}\right|= & \left|\overline{H_{1}} \cup \overline{H_{2}} \cup \overline{H_{3}}\right|+\left|\overline{H_{4}} \backslash \overline{H_{4} \cap H_{1}} \cup \overline{H_{4} \cap H_{2}} \cup \overline{H_{4} \cap H_{3}}\right|+ \\
& \left|\overline{H_{5}} \backslash \overline{H_{5} \cap H_{1}} \cup \cdots \cup \overline{H_{5} \cap H_{4}}\right| \\
= & \left(3\left[\begin{array}{c}
l-1 \\
r
\end{array}\right]-2\left[\begin{array}{c}
l-2 \\
r
\end{array}\right]\right)+2\left(\left[\begin{array}{c}
l-1 \\
r
\end{array}\right]-3\left[\begin{array}{c}
l-2 \\
r
\end{array}\right]+2\left[\begin{array}{c}
l-3 \\
r
\end{array}\right]\right)=g_{3}^{(5)} .
\end{aligned}
$$

Case 2: $H_{5}$ contains $U$ but does not contain any $W_{i}$ for $i=1,2,3$. In this case, $H_{1}, H_{2}, H_{3}, H_{4}$ intersect $H_{5}$ at 4 different hyperplanes all of which contains $U$. It follows that

$$
\begin{aligned}
\left|\overline{H_{1}} \cup \cdots \cup \overline{H_{5}}\right|= & \left|\overline{H_{1}} \cup \overline{H_{2}} \cup \overline{H_{3}}\right|+\left|\overline{H_{4}} \backslash \overline{H_{4} \cap H_{1}} \cup \overline{H_{4} \cap H_{2}} \cup \overline{H_{4} \cap H_{3}}\right|+ \\
& \left|\overline{H_{5}} \backslash \overline{H_{5} \cap H_{1}} \cup \cdots \cup \overline{H_{5} \cap H_{4}}\right| \\
= & \left(3\left[\begin{array}{c}
l-1 \\
r
\end{array}\right]-2\left[\begin{array}{c}
l-2 \\
r
\end{array}\right]\right)+\left(\left[\begin{array}{c}
l-1 \\
r
\end{array}\right]-3\left[\begin{array}{c}
l-2 \\
r
\end{array}\right]+2\left[\begin{array}{c}
l-3 \\
r
\end{array}\right]\right)+ \\
& \left(\left[\begin{array}{c}
l-1 \\
r
\end{array}\right]-4\left[\begin{array}{c}
l-2 \\
r
\end{array}\right]+3\left[\begin{array}{c}
l-3 \\
r
\end{array}\right]\right)=g_{5}^{(5)} .
\end{aligned}
$$


Case 3: $H_{5}$ does not contain $U$. This is the situation of Lemma 2.2. We have

$$
\begin{aligned}
\left|\overline{H_{1}} \cup \cdots \cup \overline{H_{5}}\right|= & \left|\overline{H_{1}} \cup \cdots \cup \overline{H_{4}}\right|+\left|\overline{H_{5}} \backslash \overline{H_{5} \cap H_{1}} \cup \cdots \cup \overline{H_{5} \cap H_{4}}\right| \\
= & \left(4\left[\begin{array}{c}
l-1 \\
r
\end{array}\right]-5\left[\begin{array}{c}
l-2 \\
r
\end{array}\right]+2\left[\begin{array}{c}
l-3 \\
r
\end{array}\right]\right)+ \\
& \left(\left[\begin{array}{c}
l-1 \\
r
\end{array}\right]-4\left[\begin{array}{c}
l-2 \\
r
\end{array}\right]+5\left[\begin{array}{c}
l-3 \\
r
\end{array}\right]-2\left[\begin{array}{c}
l-4 \\
r
\end{array}\right]\right)=g_{4}^{(5)} .
\end{aligned}
$$

- If $x(\mathcal{H})=2$, then $W=H_{1} \cap H_{2} \cap H_{3}$ has dimension $l-3$. The formula for $g_{6}^{(5)}$ comes from the case when $H_{4}$ and $H_{5}$ both contain $W ; g_{7}^{(5)}$ is obtained when $W \subset H_{4}$ but $W \not \subset H_{5}$ or vice versa; $g_{8}^{(5)}$ is obtained when $W$ is neither a subspace of $H_{4}$ or $H_{5}$ and $H_{5}$ does not contain the intersection $U=H_{1} \cap H_{2} \cap H_{3} \cap H_{4}$, and $g_{9}^{(5)}$ is obtained when $W$ is neither a subspace of $H_{4}$ nor $H_{5}$, yet $H_{5}$ does contain $U$. The computation is similar to the previous case.

\subsection{Tighter bounds and the packing arrangement}

We first consider the simplest case when $r=1$. The total number of lines (i.e. 1-dimensional subspaces) of a $k$-dimensional vector space $S$ over $\mathbb{F}_{q}$ is

$$
\left[\begin{array}{l}
k \\
1
\end{array}\right]=[k]=\frac{q^{k}-1}{q-1} .
$$

Let $V$ be any $k$-2-dimensional subspace of $S$, and $H_{1}, \ldots, H_{q+1}$ be the set of all hyperplanes containing $V$. Then, the inclusion-exclusion formula (3) gives

$$
\left|\overline{H_{1}} \cup \cdots \cup \overline{H_{q+1}}\right|=(q+1)\left[\begin{array}{c}
k-1 \\
1
\end{array}\right]-q\left[\begin{array}{c}
k-2 \\
1
\end{array}\right]=(q+1) \frac{q^{k-1}-1}{q-1}-q \frac{q^{k-2}-1}{q-1}=\frac{q^{k}-1}{q-1} .
$$

The following theorem follows immediately.

Theorem 2.5. When $r=1$ and $d \geq q+1$, the maximum value of $\left|\overline{H_{1}} \cup \cdots \cup \overline{H_{d}}\right|$ is exactly $[k]$, the total number of lines in $S$. One way to obtain this maximum is to have $q+1$ of the hyperplanes contain $a(k-2)$-dimensional subspace of $S$.

For the rest of this section, we can assume $r \geq 2$. We first give a particular arrangement called the packing arrangement which proves to be optimal in certain cases.

Definition 2.6 (Packing Arrangement). Suppose $1+q<d \leq 1+q+q^{2}$. Let $S$ be the $k$-dimensional vector space that the hyperplanes belong to. Let $V$ be any $(k-2)$-dimensional subspace of $S$, and $W$ be any $(k-3)$-dimensional subspace of $V$. The packing arrangement of $d$ hyperplanes is an arrangement in which $q+1$ hyperplanes, say $H_{1}, \ldots, H_{q+1}$, all contain $V$ and the rest of the hyperplanes contain $W$.

We could define the packing arrangement for larger values of $d$. However, for the purposes of this paper $d \leq 1+q+q^{2}$ is sufficient. The following lemma tells us the "cost" of this arrangement.

Lemma 2.7. Consider $1+q<d \leq 1+q+q^{2}$, and let $H_{1}, \ldots, H_{d}$ be in the packing configuration. Then,

$$
\left|\overline{H_{1}} \cup \cdots \cup \overline{H_{d}}\right|=d\left[\begin{array}{c}
k-1 \\
r
\end{array}\right]-\left(d(q+1)-\left(1+q+q^{2}\right)\right)\left[\begin{array}{c}
k-2 \\
r
\end{array}\right]+q(d-q-1)\left[\begin{array}{c}
k-3 \\
r
\end{array}\right] .
$$


Proof. Without loss of generality, assume $H_{1}, \ldots, H_{q+1}$ intersect at a $(k-2)$-dimensional subspace $V$ and the rest of the hyperplanes contain a $(k-3)$-dimensional subspace $W \subset V$. Consider any $H_{i}$ where $q+1<i \leq d$. Let $V_{j}=H_{i} \cap H_{j}$, for $j \in\{1, \ldots, i-1\}$. Note that all $V_{j}$ contain $W$; and, due to Lemma 2.2 it is easy to see that $V_{1}, \ldots, V_{q+1}$ are different hyperplanes of $H_{i}$. Moreover, the total number of hyperplanes in $H_{i}$ that contain $W$ is exactly $1+q$. Hence,

$$
\left\{V_{1}, \ldots, V_{q+1}\right\}=\left\{V_{1}, \ldots, V_{i-1}\right\} .
$$

Consequently,

$$
\begin{aligned}
\left|\overline{H_{i}} \backslash \overline{H_{1}} \cup \cdots \cup \overline{H_{i-1}}\right| & =\left|\overline{H_{i}} \backslash \overline{H_{1} \cap H_{i}} \cup \cdots \cup \overline{H_{i-1} \cap H_{i}}\right| \\
& =\left|\overline{H_{i}} \backslash \overline{V_{1}} \cup \cdots \cup \overline{V_{i-1}}\right| \\
& =\left|\overline{H_{i}} \backslash \overline{V_{1}} \cup \cdots \cup \overline{V_{q+1}}\right| \\
& =\left|\overline{H_{i}}\right|-\left|\overline{V_{1}} \cup \cdots \cup \overline{V_{q+1}}\right| \\
& =\left[\begin{array}{c}
k-1 \\
r
\end{array}\right]-(q+1)\left[\begin{array}{c}
k-2 \\
r
\end{array}\right]+q\left[\begin{array}{c}
k-3 \\
r
\end{array}\right] .
\end{aligned}
$$

Finally,

$$
\begin{aligned}
\left|\bigcup_{i=1}^{d} \overline{H_{i}}\right| & =\left|\bigcup_{i=1}^{q+1} \overline{H_{i}}\right|+\sum_{i=q+2}^{d}\left|\overline{H_{i}} \backslash \overline{H_{1}} \cup \cdots \cup \overline{H_{i-1}}\right| \\
& =(q+1)\left[\begin{array}{c}
k-1 \\
r
\end{array}\right]-q\left[\begin{array}{c}
k-2 \\
r
\end{array}\right]+(d-q-1)\left(\left[\begin{array}{c}
k-1 \\
r
\end{array}\right]-(q+1)\left[\begin{array}{c}
k-2 \\
r
\end{array}\right]+q\left[\begin{array}{c}
k-3 \\
r
\end{array}\right]\right) \\
& =d\left[\begin{array}{c}
k-1 \\
r
\end{array}\right]-\left(d(q+1)-\left(1+q+q^{2}\right)\right)\left[\begin{array}{c}
k-2 \\
r
\end{array}\right]+q(d-q-1)\left[\begin{array}{c}
k-3 \\
r
\end{array}\right] .
\end{aligned}
$$

Theorem 2.8. Suppose $d \leq[k]$. Consider any $d$ hyperplanes $H_{1}, \ldots, H_{d}$ of a $k$-dimensional vector space $S$ over $\mathbb{F}_{q}$. Let $x$ be a maximal number of hyperplanes intersecting in a $(k-2)$-dimensional subspace $V \subset C$. Then,

$$
\left|\overline{H_{1}} \cup \cdots \cup \overline{H_{d}}\right| \leq d\left[\begin{array}{c}
k-1 \\
r
\end{array}\right]+\left[\begin{array}{c}
k-2 \\
r
\end{array}\right]-d\left[\begin{array}{c}
k-3 \\
r
\end{array}\right]+x(x-d-1)\left(\left[\begin{array}{c}
k-2 \\
r
\end{array}\right]-\left[\begin{array}{c}
k-3 \\
r
\end{array}\right]\right)
$$

Proof. Without loss of generality, assume $H_{1}, \ldots, H_{x}$ intersect at $V$ of dimension $(k-2)$, and no other $H_{i}$ contains $V$. We invoke Lemmas 2.2 and formula (3) again. Since $H_{i}$ does not contain $V$, it is easy to see that, for $1 \leq j \leq x$, the vector spaces $V_{j}=H_{i} \cap H_{j}$ are all distinct with dimension one less than $H_{j}$. Also, the intersection of the $V_{j}$ has dimension one less than $V$. It follows that

$$
\left|\overline{H_{i}} \backslash \overline{H_{1}} \cup \cdots \cup \overline{H_{x}}\right|=\left|\overline{H_{i}} \backslash \overline{V_{1}} \cup \cdots \cup \overline{V_{x}}\right|=\left[\begin{array}{c}
k-1 \\
r
\end{array}\right]-x\left[\begin{array}{c}
k-2 \\
r
\end{array}\right]+(x-1)\left[\begin{array}{c}
k-3 \\
r
\end{array}\right] .
$$

Consequently,

$$
\begin{aligned}
\left|\bigcup_{i=1}^{d} \overline{H_{i}}\right| & =\left|\bigcup_{i=1}^{x} \overline{H_{i}}\right|+\sum_{i=x+1}^{d}\left|\overline{H_{i}} \backslash \overline{H_{1}} \cup \cdots \cup \overline{H_{i-1}}\right| \\
& \leq\left|\bigcup_{i=1}^{x} \overline{H_{i}}\right|+\sum_{i=x+1}^{d}\left|\overline{H_{i}} \backslash \overline{H_{1}} \cup \cdots \cup \overline{H_{x}}\right| \\
& =x\left[\begin{array}{c}
k-1 \\
r
\end{array}\right]-(x-1)\left[\begin{array}{c}
k-2 \\
r
\end{array}\right]+(d-x)\left(\left[\begin{array}{c}
k-1 \\
r
\end{array}\right]-x\left[\begin{array}{c}
k-2 \\
r
\end{array}\right]+(x-1)\left[\begin{array}{c}
k-3 \\
r
\end{array}\right]\right) \\
& =d\left[\begin{array}{c}
k-1 \\
r
\end{array}\right]+\left[\begin{array}{c}
k-2 \\
r
\end{array}\right]-d\left[\begin{array}{c}
k-3 \\
r
\end{array}\right]+x(x-d-1)\left(\left[\begin{array}{c}
k-2 \\
r
\end{array}\right]-\left[\begin{array}{c}
k-3 \\
r
\end{array}\right]\right)
\end{aligned}
$$


We get Theorem 2.1 for free.

Corollary 2.9 (Same as Theorem 2.1). Suppose $2 \leq d \leq q+1$. Then, for any $d$ hyperplanes $H_{1}, \ldots, H_{d}$ of a $k$-dimensional vector space $C$ over $\mathbb{F}_{q}$ we have

$$
\left|\overline{H_{1}} \cup \cdots \cup \overline{H_{d}}\right| \leq d\left[\begin{array}{c}
k-1 \\
r
\end{array}\right]-(d-1)\left[\begin{array}{c}
k-2 \\
r
\end{array}\right] .
$$

Moreover, there exists an arrangement of hyperplanes achieving the right hand side.

Proof. Without loss of generality, suppose $H_{1}, \ldots, H_{x}$ intersect at some $(k-2)$-dimensional subspace $V$, and no other $H_{i}$ contains $V$. Note that $2 \leq x \leq d$. Thus $x(x-d-1) \leq-d$. The triangle identity (6) gives

$$
\left[\begin{array}{c}
k-2 \\
r
\end{array}\right]-\left[\begin{array}{c}
k-3 \\
r
\end{array}\right]=q^{k-2-r}\left[\begin{array}{l}
k-3 \\
r-1
\end{array}\right] \geq 0 .
$$

Relation (21) implies

$$
\begin{aligned}
\left|\overline{H_{1}} \cup \cdots \cup \overline{H_{d}}\right| & \leq d\left[\begin{array}{c}
k-1 \\
r
\end{array}\right]+\left[\begin{array}{c}
k-2 \\
r
\end{array}\right]-d\left[\begin{array}{c}
k-3 \\
r
\end{array}\right]-d\left(\left[\begin{array}{c}
k-2 \\
r
\end{array}\right]-\left[\begin{array}{c}
k-3 \\
r
\end{array}\right]\right) \\
& =d\left[\begin{array}{c}
k-1 \\
r
\end{array}\right]-(d-1)\left[\begin{array}{c}
k-2 \\
r
\end{array}\right] .
\end{aligned}
$$

The inequality is tight because equality can be obtained by choosing $d$ hyperplanes $H_{1}, \ldots, H_{d}$ all of which contain a $(k-2)$-dimensional subspace $V$.

Theorem 2.10. Suppose $d \geq q+2$ and $k>r \geq 2$. Then, for any $d$ hyperplanes $H_{1}, \ldots, H_{d}$ of a $k$-dimensional vector space $S$ over $\mathbb{F}_{q}$ we have

$$
\left|\overline{H_{1}} \cup \cdots \cup \overline{H_{d}}\right| \leq d\left[\begin{array}{c}
k-1 \\
r
\end{array}\right]-(2 d-3)\left[\begin{array}{c}
k-2 \\
r
\end{array}\right]+(d-2)\left[\begin{array}{c}
k-3 \\
r
\end{array}\right] .
$$

Moreover, when $d=q+2$ the packing arrangement achieves the bound.

Proof. Without loss of generality, suppose $H_{1}, \ldots, H_{x}$ intersect at some $(k-2)$-dimensional subspace $V$, and no other $H_{i}$ contains $V$. Note that, in this case $2 \leq x \leq q+1$, and thus $x(x-d-1) \leq$ $2(2-d-1)=-2(d-1)$. Relation (21) implies

$$
\begin{aligned}
\left|\overline{H_{1}} \cup \cdots \cup \overline{H_{d}}\right| & \leq d\left[\begin{array}{c}
k-1 \\
r
\end{array}\right]+\left[\begin{array}{c}
k-2 \\
r
\end{array}\right]-d\left[\begin{array}{c}
k-3 \\
r
\end{array}\right]-2(d-1)\left(\left[\begin{array}{c}
k-2 \\
r
\end{array}\right]-\left[\begin{array}{c}
k-3 \\
r
\end{array}\right]\right) \\
& =d\left[\begin{array}{c}
k-1 \\
r
\end{array}\right]-(2 d-3)\left[\begin{array}{c}
k-2 \\
r
\end{array}\right]+(d-2)\left[\begin{array}{c}
k-3 \\
r
\end{array}\right] .
\end{aligned}
$$

When $d=q+2$, we only have to verify that the right hand side of (23) is the same as that of (20), which is mechanical.

Theorem 2.11. Suppose $d \geq q+3$ and $k>r \geq 2$. Then, for any $d$ hyperplanes $H_{1}, \ldots, H_{d}$ of $a$ $k$-dimensional vector space $S$ over $\mathbb{F}_{q}$ we have

$$
\left|\overline{H_{1}} \cup \cdots \cup \overline{H_{d}}\right| \leq d\left[\begin{array}{c}
k-1 \\
r
\end{array}\right]-(3 d-7)\left[\begin{array}{c}
k-2 \\
r
\end{array}\right]+(2 d-6)\left[\begin{array}{c}
k-3 \\
r
\end{array}\right] .
$$

Moreover, when $d=q+3$ the packing arrangement achieves the bound. 
Proof. Let $x=x\left(\left\{H_{1}, \ldots, H_{d}\right\}\right)$. Without loss of generality, suppose $H_{1}, \ldots, H_{x}$ intersect at some $(k-2)$-dimensional subspace $V$, and no other $H_{i}$ contains $V$. Consider two cases as follows.

Case 1: $x \geq 3$. Note that, $x \leq q+1 \leq d-2$, and thus $x(x-d-1) \leq 3(3-d-1)=-3(d-2)$. Relation (21) implies

$$
\begin{aligned}
\left|\overline{H_{1}} \cup \cdots \cup \overline{H_{d}}\right| & \leq d\left[\begin{array}{c}
k-1 \\
r
\end{array}\right]+\left[\begin{array}{c}
k-2 \\
r
\end{array}\right]-d\left[\begin{array}{c}
k-3 \\
r
\end{array}\right]-3(d-2)\left(\left[\begin{array}{c}
k-2 \\
r
\end{array}\right]-\left[\begin{array}{c}
k-3 \\
r
\end{array}\right]\right) \\
& =d\left[\begin{array}{c}
k-1 \\
r
\end{array}\right]-(3 d-7)\left[\begin{array}{c}
k-2 \\
r
\end{array}\right]+(2 d-6)\left[\begin{array}{c}
k-3 \\
r
\end{array}\right] .
\end{aligned}
$$

Case 2: $x=2$. Applying Lemma 2.3 with $l=k$ we get

$$
\left|\overline{H_{1}} \cup \overline{H_{2}} \cup \overline{H_{3}} \cup \overline{H_{4}}\right| \leq \max \left\{g_{3}^{(4)}, g_{4}^{(4)}\right\}=g_{3}^{(4)}=4\left[\begin{array}{c}
k-1 \\
r
\end{array}\right]-6\left[\begin{array}{c}
k-2 \\
r
\end{array}\right]+4\left[\begin{array}{c}
k-3 \\
r
\end{array}\right]-\left[\begin{array}{c}
k-4 \\
r
\end{array}\right] .
$$

Consider any $H_{i}$ with $i>4$. For $j=1,2,3$, 4 , let $V_{j}=H_{i} \cap H_{j}$. Then, the $V_{j}$ are four different hyperplanes of $H_{i}$, because $x=2$. Applying Lemma 2.3 with $l=k-1$ we get

$$
\left|\overline{V_{1}} \cup \overline{V_{2}} \cup \overline{V_{3}} \cup \overline{V_{4}}\right| \geq \min \left\{g_{1}^{(4)}, g_{2}^{(4)}, g_{3}^{(4)}, g_{4}^{(4)}\right\}=g_{4}^{(4)}=4\left[\begin{array}{c}
k-2 \\
r
\end{array}\right]-6\left[\begin{array}{c}
k-3 \\
r
\end{array}\right]+3\left[\begin{array}{c}
k-4 \\
r
\end{array}\right] .
$$

Hence,

$$
\left|\overline{H_{i}} \backslash \overline{H_{1}} \cup \overline{H_{2}} \cup \overline{H_{3}} \cup \overline{H_{4}}\right|=\left|\overline{H_{i}}\right|-\left|\overline{V_{1}} \cup \overline{V_{2}} \cup \overline{V_{3}} \cup \overline{V_{4}}\right| \leq\left[\begin{array}{c}
k-1 \\
r
\end{array}\right]-4\left[\begin{array}{c}
k-2 \\
r
\end{array}\right]+6\left[\begin{array}{c}
k-3 \\
r
\end{array}\right]-3\left[\begin{array}{c}
k-4 \\
r
\end{array}\right] .
$$

Putting them all together, we get

$$
\begin{aligned}
\left|\bigcup_{i=1}^{d} \overline{H_{i}}\right|= & \left|\overline{H_{1}} \cup \overline{H_{2}} \cup \overline{H_{3}} \cup \overline{H_{4}}\right|+\sum_{i=5}^{d}\left|\overline{H_{i}} \backslash \overline{H_{1}} \cup \overline{H_{2}} \cup \overline{H_{3}} \cup \overline{H_{4}}\right| \\
\leq & \left(4\left[\begin{array}{c}
k-1 \\
r
\end{array}\right]-6\left[\begin{array}{c}
k-2 \\
r
\end{array}\right]+4\left[\begin{array}{c}
k-3 \\
r
\end{array}\right]-\left[\begin{array}{c}
k-4 \\
r
\end{array}\right]\right)+ \\
& (d-4)\left(\left[\begin{array}{c}
k-1 \\
r
\end{array}\right]-4\left[\begin{array}{c}
k-2 \\
r
\end{array}\right]+6\left[\begin{array}{c}
k-3 \\
r
\end{array}\right]-3\left[\begin{array}{c}
k-4 \\
r
\end{array}\right]\right) \\
= & d\left[\begin{array}{c}
k-1 \\
r
\end{array}\right]-(4 d-10)\left[\begin{array}{c}
k-2 \\
r
\end{array}\right]+(6 d-20)\left[\begin{array}{c}
k-3 \\
r
\end{array}\right]-(3 d-12)\left[\begin{array}{c}
k-4 \\
r
\end{array}\right]
\end{aligned}
$$

It is easy to see that, when $k>r \geq 2$ the last expression is at most the right hand side of (24).

Lastly, when $d=q+3$ the fact that the packing arrangement achieves the bound (24) is straightforward.

Theorem 2.12. Suppose $d \geq q+4$ and $k>r \geq 2$. Then, for any $d$ hyperplanes $H_{1}, \ldots, H_{d}$ of a $k$-dimensional vector space $S$ over $\mathbb{F}_{q}$ we have

$$
\left|\overline{H_{1}} \cup \cdots \cup \overline{H_{d}}\right| \leq d\left[\begin{array}{c}
k-1 \\
r
\end{array}\right]-(4 d-13)\left[\begin{array}{c}
k-2 \\
r
\end{array}\right]+(3 d-12)\left[\begin{array}{c}
k-3 \\
r
\end{array}\right] .
$$

Moreover, when $d=q+4$ the packing arrangement achieves the bound.

Proof. The proof is similar to the previous theorem with three cases to consider: $x \geq 4, x=3$, and $x=2$. This time we make use of Lemma 2.4 and its various relations. 


\section{Tighter analysis of $M_{q}(m, k, r)$}

The results of the previous section help us analyze the $M_{q}(m, k, r)$ construction. Firstly, we show that $M_{q}(m, k, 1)$ is not a good design when $d \geq q+1$. The result is a direct corollary of Theorem 2.5.

Corollary 3.1. When $d \geq q+1$, then $M_{q}(m, k, 1)$ is not d-disjunct.

Proof. Let $C_{0}$ be a $k$-dimensional subspace of $\mathbb{F}_{q}^{m}$. Let $H_{1}, \ldots, H_{q+1}$ be hyperplanes of $C_{0}$ chosen according to Theorem 2.5. Let $v$ be any vector in $\mathbb{F}_{q}^{m}$ not belonging to $C_{0}$. For $i=1, \ldots, d$, let $C_{i}=\operatorname{span}\left\{H_{i}, v\right\}$. Choose arbitrarily $k$-dimensional subspaces $C_{q+2}, \ldots, C_{d}$. Then, it is easy to see that $\overline{C_{0}} \backslash \overline{C_{1}} \cup \cdots \cup \overline{C_{d}}=\emptyset$.

Secondly, the number of columns of $M_{q}(m, k, r)$ is $\left[\begin{array}{c}m \\ k\end{array}\right]$, which is exponentially larger than $[k]$, the number of hyperplanes in a $k$-dimensional vector space. The following theorem shows a limit of the pooling design.

Theorem 3.2. If $d \geq[k]$, then $M_{q}(m, k, r)$ is not d-disjunct.

Proof. Consider any $k$-dimensional subspace $C_{0}$ of $\mathbb{F}_{q}^{m}$. Let $H_{1}, \ldots, H_{[k]}$ be the set of all hyperplanes of $C_{0}$. Let $v$ be any vector in $\mathbb{F}_{q}^{m} \backslash C_{0}$. For any $i=1, \ldots,[k]$, define $C_{i}=\operatorname{span}\left\{v, H_{i}\right\}$. For $i=$ $[k]+1, \ldots, d$, choose $k$-dimensional subspaces $C_{i}$ arbitrarily as long as they have not been chosen before. Then, $\left|\overline{C_{0}} \backslash \overline{C_{1}} \cup \cdots \cup \overline{C_{d}}\right|=0$, namely $M_{q}(m, k, r)$ is not $d$-disjunct. This is because any $r$ dimensional subspace of $C_{0}$ is also a subspace of some $H_{i}, i=1, \ldots[k]$; thus, it is also an $r$-dimensional subspace of $C_{i}$.

Henceforth, we only need to consider the case when $r \geq 2$ and $q+2 \leq d \leq[k]$. The following corollaries follow from Theorems 2.10, 2.11, and 2.12, respectively.

Corollary 3.3. When $d \geq q+2$ and $m>k>r \geq 2, M_{q}(m, k, r)$ is $d^{z}$-disjunct, where

$$
z=\left[\begin{array}{l}
k \\
r
\end{array}\right]-d\left[\begin{array}{c}
k-1 \\
r
\end{array}\right]+(2 d-3)\left[\begin{array}{c}
k-2 \\
r
\end{array}\right]-(d-2)\left[\begin{array}{c}
k-3 \\
r
\end{array}\right]
$$

Moreover, the construction is exactly $d^{z}$-disjunct when $d=q+2$.

Corollary 3.4. When $d \geq q+3$ and $m>k>r \geq 2, M_{q}(m, k, r)$ is $d^{z}$-disjunct, where

$$
z=\left[\begin{array}{l}
k \\
r
\end{array}\right]-d\left[\begin{array}{c}
k-1 \\
r
\end{array}\right]+(3 d-7)\left[\begin{array}{c}
k-2 \\
r
\end{array}\right]-(2 d-6)\left[\begin{array}{c}
k-3 \\
r
\end{array}\right]
$$

Moreover, the construction is exactly $d^{z}$-disjunct when $d=q+3$.

Corollary 3.5. When $d \geq q+4$ and $m>k>r \geq 2, M_{q}(m, k, r)$ is $d^{z}$-disjunct, where

$$
z=\left[\begin{array}{l}
k \\
r
\end{array}\right]-d\left[\begin{array}{c}
k-1 \\
r
\end{array}\right]+(4 d-13)\left[\begin{array}{c}
k-2 \\
r
\end{array}\right]-(3 d-12)\left[\begin{array}{c}
k-3 \\
r
\end{array}\right]
$$

Moreover, the construction is exactly $d^{z}$-disjunct when $d=q+4$.

Remark 3.6. Note that, to apply all the three corollaries above, we only need to find the range of $d$ which makes $z>0$. It turns out that this range is quite large, and the task is mechanical. We omit this step here. Also, it is straightforward to check that the bounds in Theorems 2.10, 2.11, and 2.12 are better than that of Theorem 2.1. 


\section{Discussions}

It is very natural to ask the converse of our hyperplane arrangement problem, leading to the following:

Problem 2 (Second Hyperplane Arrangement Problem). Given a $k$-dimensional vector space $C$ over $\mathbb{F}_{q}$, and an integer $d$ such that $1 \leq d \leq[k]$, find $d$ hyperplanes $H_{1}, \ldots, H_{d}$ of $C$ that minimizes the following quantity

$$
\left|\overline{H_{1}} \cup \cdots \cup \overline{H_{d}}\right| .
$$

Historically, there have been quite a lot of studies on hyperplane arrangements. The extremal problems such as the problem of dividing a space into as many regions as possible given a fixed number of hyperplanes are mostly on infinite vector spaces. Arrangement problems and results on finite fields mostly are about algebraic and structural information (Möbius functions, Poincaré polynomials, ...) or topological structures. The reader is referred to [23] for a good treatment of such problems. Our two hyperplane arrangement problems are new, to be best of the author's knowledge.

It is possible to show that the packing arrangement is the best for $d=q+5(q \geq 3)$ and so on, but the current method becomes too tedious to be useful. We conjecture that the packing arrangement is best for Problem 1 when $1+q<d \leq 1+q+q^{2}$. We also leave open Problem 2 at this point.

\section{References}

[1] M. Aigner, Searching with lies, J. Combin. Theory Ser. A, 74 (1996), pp. 43-56.

[2] G. E. Andrews, The theory of partitions, Cambridge University Press, Cambridge, 1998. Reprint of the 1976 original.

[3] D. J. Balding, W. J. Bruno, E. Knill, And D. C. Torney, A comparative survey of non-adaptive pooling designs, in Genetic mapping and DNA sequencing (Minneapolis, MN, 1994), Springer, New York, 1996, pp. 133-154.

[4] D. J. Balding And D. C. ToRney, Optimal pooling designs with error detection, J. Combin. Theory Ser. A, 74 (1996), pp. 131-140.

[5] A. E. F. Clementi, A. Monti, and R. Silvestri, Selective families, superimposed codes, and broadcasting on unknown radio networks (extended abstract), in Proceedings of the Twelfth Annual ACM-SIAM Symposium on Discrete Algorithms (Washington, DC, 2001), Philadelphia, PA, 2001, SIAM, pp. 709-718.

[6] A. DE BONIS AND U. VACCARO, Efficient constructions of generalized superimposed codes with applications to group testing and conflict resolution in multiple access channels, in Algorithms-ESA 2002, vol. 2461 of Lecture Notes in Comput. Sci., Springer, Berlin, 2002, pp. 335-347.

[7] - Constructions of generalized superimposed codes with applications to group testing and conflict resolution in multiple access channels, Theoret. Comput. Sci., 306 (2003), pp. 223-243.

[8] D.-Z. Du, F. Hwang, W. WU, AND T. ZnATI, New construction for transversal design, Journal of Computational Biology, 13 (2006), pp. 990-995.

[9] D.-Z. Du And F. K. Hwang, Combinatorial group testing and its applications, World Scientific Publishing Co. Inc., River Edge, NJ, 1993.

[10] A. D'Yachkov, F. Hwang, A. Macula, P. Vilenkin, and C. W. Weng, A construction of pooling designs with some happy surprises, Journal of Computational Biology, 12 (2005), pp. 1129-1136.

[11] A. G. D'YAChKOV, A. J. MACUlA, AND V. V. RYKOV, New applications and results of superimposed code theory arising from the potentialities of molecular biology, in Numbers, information and complexity (Bielefeld, 1998), Kluwer Acad. Publ., Boston, MA, 2000, pp. 265-282.

[12] A. G. D'yachiov, A. J. Macula, JR., And V. V. RYKov, New constructions of superimposed codes, IEEE Trans. Inform. Theory, 46 (2000), pp. 284-290.

[13] A. G. D'yAchKov And V. V. RYKov, A survey of superimposed code theory, Problems Control Inform. Theory/Problemy Upravlen. Teor. Inform., 12 (1983), pp. 229-242. 
[14] W. H. KaUtz And R. C. Singleton, Nonrandom binary superimposed codes, IEEE Trans. Inf. Theory, 10 (1964), pp. 363-377.

[15] H. K. Kim And V. Lebedev, On optimal superimposed codes, J. Combin. Des., 12 (2004), pp. $79-91$.

[16] E. Knill, W. J. BRuno, And D. C. ToRney, Non-adaptive group testing in the presence of errors, Discrete Appl. Math., 88 (1998), pp. 261-290.

[17] X. MA And L. PING, Coded modulation using superimposed binary codes, IEEE Trans. Inform. Theory, 50 (2004), pp. 3331-3343.

[18] A. J. Macula, A simple construction of d-disjunct matrices with certain constant weights, Discrete Math., 162 (1996), pp. 311-312.

[19] — Error-correcting nonadaptive group testing with d $d^{e}$-disjunct matrices, Discrete Appl. Math., 80 (1997), pp. 217222.

[20] S. MUthukrishnan, On optimal strategies for searching in presence of errors, in Proceedings of the Fifth Annual ACM-SIAM Symposium on Discrete Algorithms (Arlington, VA, 1994), New York, 1994, ACM, pp. 680-689.

[21] H. Q. NGO AND D.-Z. DU, A survey on combinatorial group testing algorithms with applications to DNA library screening, in Discrete mathematical problems with medical applications (New Brunswick, NJ, 1999), vol. 55 of DIMACS Ser. Discrete Math. Theoret. Comput. Sci., Amer. Math. Soc., Providence, RI, 2000, pp. 171-182.

[22] - New constructions of non-adaptive and error-tolerance pooling designs, Discrete Math., 243 (2002), pp. 161-170.

[23] P. Orlik And H. Terao, Arrangements of hyperplanes, vol. 300 of Grundlehren der Mathematischen Wissenschaften [Fundamental Principles of Mathematical Sciences], Springer-Verlag, Berlin, 1992.

[24] J. K. Percus, O. E. Percus, W. J. Bruno, And D. C. Torney, Asymptotics of pooling design performance, J. Appl. Probab., 36 (1999), pp. 951-964.

[25] J. H. VAn Lint And R. M. Wilson, A course in combinatorics, Cambridge University Press, Cambridge, second ed., 2001.

[26] J. K. WOLF, Born again group testing: multiaccess communications, IEEE Transaction on Information Theory, IT-31 (1985), pp. 185-191. 